\title{
The Design of Intelligent Greenhouse Distributed Measurement and Control System Based on ARM
}

\author{
Dan Li \\ College of Electrical and Information Engineering \\ Jilin Agricultural Science and Technology College \\ Jilin City, China \\ 653301981@qq.com
}

\begin{abstract}
With the rapid development of agricultural modernization, intelligent greenhouse becomes more and more popular. This paper describes one kind of distributed measurement and control system for intelligent greenhouse environmental parameters. Every greenhouse measurement and control terminals based on ARM microprocessor get sensor measurements of environmental parameters through the serial bus, and drive the servo machine to control the environmental parameters. Server communicates with the greenhouse terminals through the distributed network, in order to realize that only one central computer can measure and control every greenhouse environment factors in real-time. This system in laboratory environment has precise and stable operation.
\end{abstract}

Keywords-intelligent greenhouse; distributed network; ARM microprocessor

\section{INTRODUCTION}

With the rapid development of agricultural modernization, we not only take advantages of natural resources, but also environmental factors to get the best condition of crop growth, such as temperature, humidity and light which may be controlled by intelligent greenhouse distributed measurement and control system. The best condition can increase crop yield, improve quality, regulate the growth period, and improve the economic efficiency. It is very important to measure and control environmental factors timely and accurately. The modern intelligent greenhouse is the prevalent development of greenhouse, because of its energy saving, environmental protection and improving the working conditions. Therefore, it is very meaningful and important that this paper deals with the hardware structure and software algorithm problems of intelligent greenhouse distributed measurement and control system. [1]

In order to measure and control the greenhouse temperature accurately and in real time, and in order to research greenhouse temperature effects on agricultural production more easily, this paper uses the technology of single-chip microcomputer and digital sensor technology, to design and develop intelligent greenhouse distributed measurement and control system based on S3C44B0X microprocessor.

\section{HIGH LEVEL DESIGN OF SYSTEM PLATFORM}

One machine, which is a common temperature instrument in many markets, can only measure one location temperature at the same time. In order to show the results are universal, it is common to frequently change the position measured by the machine. To solve the problem, this paper makes only one intelligent greenhouse distributed measurement and control system with many temperature detection headers. High precision digital temperature sensor of each detection header can communicate with the central control unit through the serial bus. [2]

The temperature parameters value, which measured by intelligent greenhouse distributed measurement and control system, can be displayed on the LED Seven-Segment digital tubes in real-time, or can be stored in the system's internal part, also can be directly obtained through the distributed network by server computer, for more complex data analysis operations and data printing. Intelligent greenhouse can automatically control servo actuators according to the crops requirements of environmental factors and the current environmental factors true value. High level system platform design as shown in Figure1:

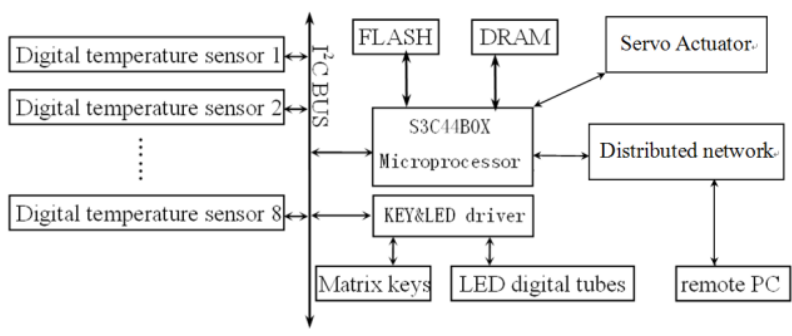

Figure1. High level system platform diagram

\section{THE DESIGN OF APPLICATION SOFTWARE}

The system server connects to the RS-485 distributed communications network by serial interface, in order to measure and control every greenhouse test point in real time. Application software running on the server is intelligent greenhouse distributed measurement and control software, which is composed by the Visual Basic language. Its user interface is shown in the Figure 2. Software mainly consists of five parts. The first part function is to select the server serial interface number, connected to a distributed network. The second part function is to select the greenhouse number, which is distributed terminal number. The third part function is to select the greenhouse environmental factors sensor number. The fourth part function is to display the current 
measured values. The fifth part function is manually to control servo actuator.

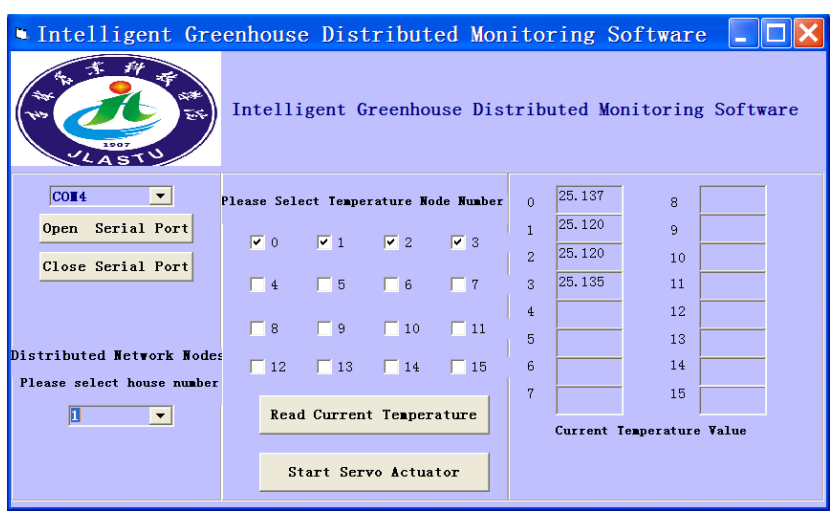

Figure2. Application software user interface

\section{THE DESIGN OF DISTRIBUTED NETWORK}

This system uses the RS-485 bus to build the distributed network. RS-485 standard serial communication interface provides balanced differential signals transmission, has strong ability to resist common-mode interference, and achieves greater transmission distance and more transmission rate. While the bus has stronger driving ability, it can hang up 32 bus transceiver to form a network at the same time. RS-232 standard used in server serial interface is different from RS-485 bus signal logic level definition used in distributed network. For this reason, while the computer system sends and receives the data frame of the distributed network, it is necessary to convert the signal logic level. This system uses MAX232 and MAX485 chips to complete the level matching. [3]

Distributed network upper layer communication protocol adopts the MODBUS protocol, whose data frame format is "the address + the function code + the amount of data + data1... + data N + CRC high byte + CRC low byte".

\section{THE DESIGN OF DISTRIBUTED TERMINALS}

\section{A. The Design of Central Control Module}

In order to improve data processing capability of intelligent greenhouse distributed measurement and control system, and to upgrade its performance to the high-end temperature measurement instrument, this paper uses Samsung's high performance-to-price ratio and high performance 16/32 bit RISC microprocessor S3C44B0X chips. S3C44B0X uses ARM7TDMI core, 0.25 um CMOS standard unit memory. S3C44B0X also provides the following parts: $8 \mathrm{~KB}$ Cache, optional internal SRAM, 2 channels UART, 4 channel DMA, 8 channels of the 12 bit ADC channels, the I2C/I2S bus interface, synchronous SIO interface and pairs of clock PLL, and other resources. [4]

Because it does not have internal FLASH, S3C44B0X puts FLASH Chip MBM29LV160T into the system. According to the intrinsic characteristics of the S3C44B0X, it takes Pin nGCSO as a FLASH optional port to make FLASH addresses start from 0X00000000.

The system uses HY57V641620 chip as external DRAM of S3C44B0X. According to the intrinsic characteristics of the S3C44B0X, it takes Pin MGCS6 as a DRAM optional port to make DRAM addresses start from 0X0C000000.

\section{B. The Design of Temperature Measurement Module}

In order to measure temperatures in many locations simultaneously, the design should put several pieces of temperature sensors on the serial communication bus. In order to achieve high accuracy of temperature monitoring, the design should choose the digital temperature sensor with 8-bit or more bits resolution. In view of the above reasons, this paper select United States Analog Devices (ADI) singlechip digital temperature sensor AD7416. The sensor contains a band gap temperature sensor and a 10-bit ADC, which can convert analog temperature value to digital signal with quantitative intervals of $0.25^{\circ} \mathrm{C}$.Its On-chip registers can be programmed with high and low temperature limits, and an open drain Over-Temperature Indicator(OTI) output is provided, which becomes active when a programmed limit is exceeded. Furthermore, an $\mathrm{I}^{2} \mathrm{C}$-compatible serial interface allows the AD7416 registers to be written to and read back; up to eight AD7416 can be connected to a single bus. Specific connection circuit as shown in Figure 3:

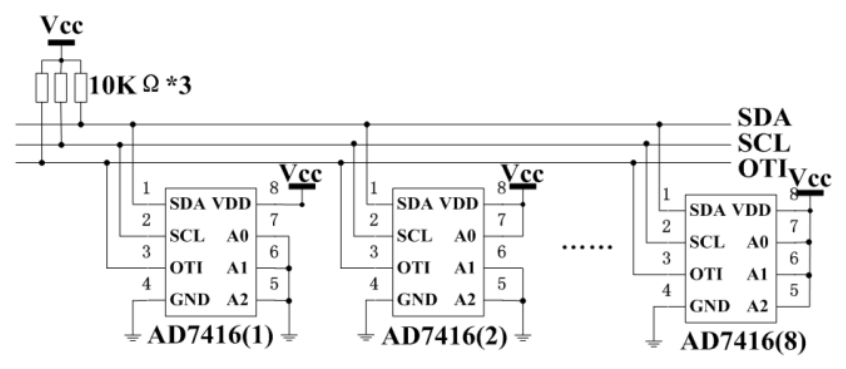

Figure3. $\mathrm{I}^{2} \mathrm{C}$ serial bus interface circuit for $\mathrm{AD} 7416$

In the interface circuit, the $\mathrm{AD} 7416$ carries out the $\mathrm{I}^{2} \mathrm{C}$ serial bus physical properties by pins SCL and SDA which are connected to pull-up resistor. AD7416 Pin A0, A1, A2 are chip selecting address lines, each of which can be set to the high level or low, so it can get eight different kinds of state through the combination. Therefore, selectable serial bus address allows connection of up to eight AD7416 to a single $\mathrm{I}^{2} \mathrm{C}$ serial bus. [5]

\section{The design of servo actuator interface module}

The regulation of greenhouse environmental factors is completed by the servo actuators, which are driven by the distributed terminals. Intelligent greenhouse can automatically control servo actuators according to the crops requirements of environmental factors and the current environmental factors true value. The interface between the system terminals and servo actuators is completed by solid state relays. The system uses ARM microprocessor GPIO pins to control solid state relays open or close, so as to 
realize servo actuators stop or start, and to complete the closed-loop control of greenhouse environmental factors. Of course, artificial regulation of environmental factors is completed by the fifth part function of the application software on the server.

\section{The Design of Interface Module}

Interface design mainly includes: design of keys and LED digital tubes interface, then design of interface between the system and the distributed network. This paper discusses mainly the latter design.

S3C44B0X chip provides 2 serial UART channels. To get data transmission between the greenhouse temperature measurement and control system and server computer, this paper uses UART0 as asynchronous serial communication interface, which is connected to the distributed network.

S3C44B0X chip's UART0 interface uses TTL-level, which is expressed in high and low level voltage state. However, server computer asynchronous serial communication interface uses RS-232C level, which is positive or negative voltage to represent logical state. In order to correctly match the electrical characteristics, it must deal with the voltage level and logical relationship between RS-232C and TTL circuits. For this purpose, UART0 serial interface circuit uses MAX3232 chip to deal with the voltage level and logical relationship transformation. The communication hardware connection circuit between system and the master PC serial interface is shown in Figure 4:

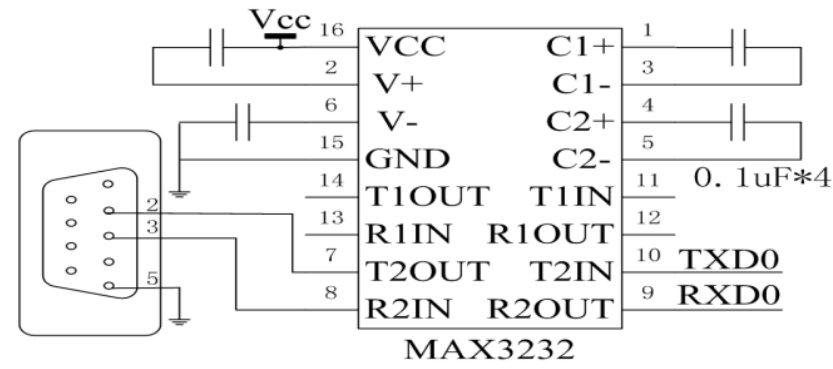

Figure4. UART0 serial interface circuit

In UART0 serial interface circuit, the data issued by S3C44B0X chip's TXD0 pin is transferred from MAX3232 chip to the server computer with RS-232C level. At the same time, PC's data is transferred from MAX3232 chip to S3C44B0X chip RXD0 PIN with TTL level.

\section{PORTING UCOS-II TO ARM MICROPROCESSOR}

uCOS-II is the real-time operating system kernel which was first published in 1992. It is used in all kinds of applications such as cameras, medical instruments, ATM machines, industrial robots, and many more.

\section{A. $\quad$ uCOS-II File System}

uCOS-II file system structure is showed in Figure 5, in which application software layer is based on the uCOS-II code. uCOS-II includes three parts: The first part is core code, which has nothing to do with the processor. This part includes seven source code files and a header file. The function of the seven source code files are respectively the core management, event management, message queues management, memory management, message management, semaphores management, task scheduling and time management. The second part is configuration code, including two header files which are used to control the number of event control blocks and whether containing news management relevant code, etc. The third part is processor porting section, which includes a header file, an assembly file and a code file.

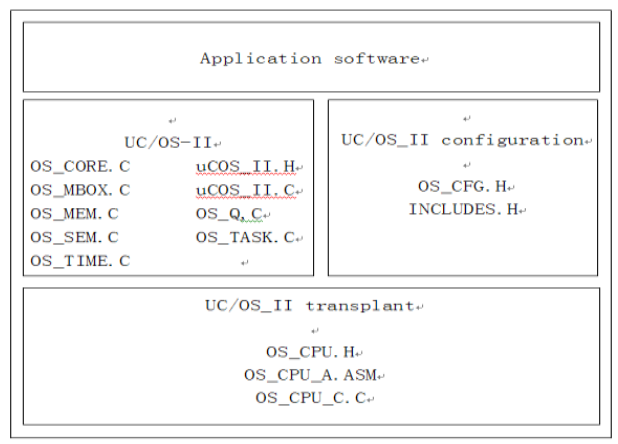

Figure5 uCOS-II Software Architecture

\section{B. Porting $\mu$ C/OS-II Procedure}

\section{1) Basic configuration and definition}

The entire basic configuration and define are included in the os_cpu.h header file. Because different microprocessors have different word length, the port of $\mu \mathrm{C} / \mathrm{OS}-\mathrm{II}$ includes a series of type definitions that ensures portability. Specifically, $\mu \mathrm{C} / \mathrm{OS}-\mathrm{II}$ 's code never makes use of C's short, int and, long data types because they are inherently nonportable. The INT16U data type, which is defined by $\mu \mathrm{C} / \mathrm{OS}$-II, always represents a 16-bit unsigned integer. However, $\mu \mathrm{C} / \mathrm{OS}$-II port to a 32-bit ARM processor could mean that an INT16U is actually declared as an unsigned short instead of an unsigned int.

2) Porting Assembly code file $O S \_C P U \_A . A S M$

OS_CPU_A.ASM file has four Assembly functions to port. In this paper, it is mainly talked about the first two functions.

The first Assembly function is OSStarHighRdy function, which is called by OSStart() to start the highest priority task ready-to-run. OSStartHighRdy() assumes that OSTCBHighRdy points to the task control block of the task with the highest priority. To run the highest priority task, it is needed to restore all processor registers from the task's stack in the proper order and, execute a return from

Interrupt. To simplify things, the stack pointer is always stored at the beginning of the task control block.

The second Assembly function is OSCtxSw function. This function is called by the macro OS_TASK_SW. OS_TASK_SW is called by the OSSched function. OSSched function is responsible for tasks switching. The OSCtxSw 
function of the OSSched function is responsible for saving the current task processor registers to the stack, and restoring processor registers from the stack for the task recovery.

3) Porting standard c code file $O S_{-} C P U \_C . C$

OS_CPU_C.C file has six fairly simple $C$ functions to port, such as OSTaskStkInit()、OSTaskCreateHook() 、

OSTaskDelHook() 、 OSTaskSwHook() 、

OSTaskStatHook() 、 OSTimeTickHook(). The only function that is actually necessary is OSTaskStkInit(). The other five functions may be declared but don't need to contain any code inside them.

\section{SUMMARY}

Intelligent greenhouse distributed measurement and control system, discussed above in this paper, uses high speed and low power ARM microprocessors instead of ordinary low speed MCS-51 series single-chip for high speed data processing and a number of external interrupt response in real time. It also uses high precision digital temperature sensor to get precise measurement temperature, which improves temperature measurement instrument performance. For this later product development and product upgrade, it may consider providing several kinds of human-computer interfaces between the temperature measurement terminal and the PC. The project discussed above in this paper is both the "Jilin City science and technology development plan fund project" and the "Jilin agricultural science and technology university scientific research fund project". This system obtains Jilin City science and technology achievements (certificate no. 2013096).

\section{REFERENCES}

[1] Wanzheng Ma, Wanmin Ma. Status and Development Research of Intelligent Greenhouse Environment Control[J]. Northern Horticulture, 2011, 23:9-11.

[2] Peng Xie, Wei Shi. Remote Monitoring and Control System Design Based on S3C2440[J]. Software,2011,11:10-11.

[3] Peng Na.Achievement of master-slave communication architecture based on RS-485[J]. Journal of Yunnan University,2007,29:259-262.

[4] Ligong Zhou. ARM and Embedded System Foundation Course[M]. Guangzhou: Guangzhou ucos MCU Development Co Ltd, 2005.

[5] Rui Shen, Haixun Yu. Temperature Acquisition System Design Based On ARM7 and LM35 [J]. Modern Electronics Technique, 2012,6. 